\title{
A
}

\section{A influência de um programa de treinamento respiratório na qualidade vocal e função pulmonar} dos participantes do Coral Unochapecó

\author{
The influence of a training program in \\ vocal quality respiratory and lung function \\ of participants Coral Unochapecó
}

FisiSenectus. Unochapecó Ano 1, n. 2 - Jul./Dez. 2013 p. 25-33

\section{Priscila Fontana}

Fisioterapeuta, graduada pela Universidade Comunitária da Região de Chapecó(Unochapecó).

\section{Lilian Marin}

Fisioterapeuta, mestranda em Envelhecimento Humano na Universidade de Passo Fundo e docente da Universidade Comunitária da Região de Chapecó (Unochapecó).

\section{Resumo \\ Introdução: a voz é a respiração sonorizada. O sistema respiratório funciona como um ativador da voz. O conhecimento e a atualização das técnicas vocais e respiratórias existentes e fisiologicamente mais equilibradas permitem o rendimento máximo e a longevidade da voz. Objetivo: verificar as repercussões de um programa de treinamento de fisioterapia respiratória, com o uso do Threshold® IMT, na qualidade vocal e função pulmonar dos participantes do Coral Unochapecó. Métodos: foram avaliadas a função pulmonar (manovacuometria e ventilometria) e função vocal (extensão vocal e TMF) de 16 coralistas, divididos em grupo experimental e controle, pré e pós-treinamento respiratório com Threshold® IMT realizado pelo grupo experimental por 15 sessões. Resultados: os resultados demonstram incremento após o treinamento respiratório com significância estatística em todas as variáveis analisadas de função pulmonar e vocal do grupo estudo e na comparação intergrupos, pré e pós-treinamento. Considerações finais: por meio dos resultados conclui-se que o treinamento respiratório com o incentivador Threshold $\circledast$ IMT é possível incrementar força muscular respiratória, volumes pulmonares e ainda potencializar a função vocal.}

\section{Palavras-chave}

Treinamento muscular respiratório. Função respiratória e vocal. Fisioterapia.

\section{Fisiß̌enectus}




\begin{abstract}
Introduction: the voice is sonorous breathing. The respiratory system functions as an activator of the voice. The knowledge and updating of existing respiratory and vocal techniques and more physiologically balanced allow full performance and longevity of the voice. Objective: to determine the effects of a training program for respiratory therapy, using the Threshold ${ }^{\circledR}$ IMT, vocal quality and respiratory function of participants Coral Unochapecó. Methods: we evaluated respiratory function (manometer and ventilation) and vocal function (vocal range and TMF) of 16 singers, divided into experimental and control groups, before and after the respiratory training with Threshold $\circledast$ IMT conducted by experimental group for 15 sessions. Results: the results showed an increase after the respiratory training with statistical significance in all variables of pulmonary and vocal function in study group and comparison between groups, pre and post training. Final consideration: through the results it is concluded that the respiratory training with Threshold ${ }^{\circledR}$ IMT increases muscle strength, pulmonary volumes and enhances the vocal function.
\end{abstract}

\title{
Keywords
}

Respiratory muscle training. Respiratory and vocal function. Physiotherapy.

\section{Introdução}

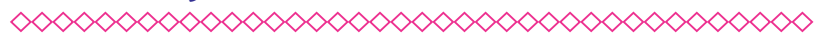

A voz é o principal instrumento de comunicação do ser humano. Apresenta um papel fundamental na expressão e relacionamento entre as pessoas. Para sua produção há necessidade de interação entre os diferentes sistemas do corpo humano, uma vez que não há um órgão específico isoladamente para produção vocal, sendo essa uma função superposta dos sistemas respiratório e digestório ${ }^{1}$.

0 sistema respiratório funciona como um ativador da voz. Do ponto de vista físico, a voz é o som básico produzido pela vibração das pregas vocais na passagem do ar durante a expiração. 0 ar expirado corresponde à fonte desencadeadora do movimento vibratório das pregas vocais ${ }^{2}$.

Embora se tratando do mesmo aparato de produção vocal, a voz cantada difere da voz falada, pois se utiliza ajustes e adaptações conforme as exigências impostas pelo canto. Entre esses ajustes, como um dos fatores preponderantes, está o domínio respiratório. Esse domínio refere-se a um maior controle do ato respiratório, exigindo um adequado padrão respiratório, força e endurance dos músculos da respiração e ampliação da capacidade pulmonar total ${ }^{3}$.

Diversos estudos demonstram o incremento de tais potencialidades por intermédio do treinamento muscular respiratório. Uma vez que no canto a respiração é programada e exige determinadas adaptações para o melhor desempenho, desta forma o objetivo dessa pesquisa foi verificar as repercussões de um programa de treinamento de fisioterapia respiratória, com o uso do incentivador Threshold $\circledast$ IMT, na qualidade vocal e função pulmonar dos participantes do Coral Unochapecó.

\section{Materiais e métodos}

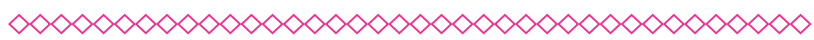

A presente pesquisa, metodologicamente caracterizada como um estudo analítico experimental, de natureza quantitativa, aprovada pelo comitê de ética em pesquisa sob protocolo de $n$. 208/08, foi desenvolvida no período de fevereiro a maio de 2009, envolvendo os participantes do Coral Unochapecó.

Foram sujeitos dessa pesquisa 16 coralistas, ambos os gêneros, com idade média de 23,5 anos, selecionados mediante critérios de inclusão e exclusão. Para serem incluídos na pesquisa os participantes deveriam fazer parte do Coral Unochapecó, bem como frequentar os ensaios com regularidade, independente do gênero, cor ou classe social, e ter lido, concordado e assinado o termo de consentimento livre e esclarecido. Foram excluídos do estudo os participantes tabagistas e portadores de doenças que acometem o sistema respiratório (asma, tuberculose, CA de pulmão etc.) e os participantes que apresentaram contraindicações à realização dos testes de função pulmonar (HAS grave, cirur- 
gias ou traumatismos recentes, hérnias abdominais, problemas de ouvido médio, glaucoma).

Os participantes foram divididos aleatoriamente em dois grupos mediante sorteio: grupo experimental e grupo controle, constituídos de igual número de participantes, ou seja, oito participantes para cada grupo. Após essa divisão, todos os participantes foram submetidos aos processos de avaliação, constituídos de duas etapas: avaliação respiratória e avaliação vocal.

A avaliação respiratória obedeceu aos critérios ditados por Costa ${ }^{4}$ e DeTurk e Cahalin ${ }^{5}$, envolvendo dados gerais de anamnese e exame físico e os testes de função pulmonar manovacuometria, com a mensuração da pressão inspiratória máxima (PEmáx) e da pressão expiratória máxima (PImáx), e ventilometria, com a medição do volume corrente (VC) e volume minuto (VM). A avaliação vocal foi realizada por profissional bacharel em canto, obedecendo aos critérios dispostos na literatura ${ }^{1,6} \mathrm{e}$ envolveu a verificação da extensão vocal e o teste do tempo máximo de fonação com as fricativas alveolares /s/ de padrão surdo e /z/ de padrão sonoro. Todos os processos de avaliação foram realizados individualmente e em ambiente fechado.

Após os processos de avaliação, os participantes do grupo controle continuaram desenvolvendo as atividades do coral, que envolvem exercícios de alongamento, respiração, aquecimento vocal, projeção, afınação e repertório. Os participantes alocados no grupo experimental, além das atividades desenvolvidas pelo coral, realizaram um treinamento respiratório com o uso do dispositivo de limiar de carga pressórica Threshold $\circledast$ IMT, da marca Respironics, iniciando com a carga de 30\% da PImáx de cada participante (seis séries de 15 ciclos), progredindo conforme a tolerância desse. As sessões ocorreram duas vezes por semana, em dias intercalado, com duração de 30 minutos, em um total de 15 sessões realizadas em grupo.

Ao final das intervenções do grupo controle, todos os participantes foram submetidos a uma reavaliação, constando dos mesmos testes já citados anteriormente.

Os resultados encontrados foram analisados estatisticamente pelo Teste t de Student pareado, na análise das variáveis intragrupos, e pelo Teste $t$ de Student, para amostras independentes na aná- lise das variáveis intergrupos, utilizando o software BioEstat 5.0 e considerado valor de significância $p \leq 0,05$.

\section{Resultados}

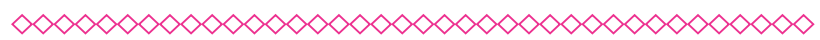

A amostra final foi composta de 16 participantes, alocados em igual número nos grupos controle e experimental. Desse total, $68,75 \%$ eram do gênero feminino e $31,25 \%$ do gênero. 0 tempo de participação no coral variou entre 1 a 11 anos, sendo a média da amostra de três anos, visto que tal variação não gerou valores de significância estatística relacionada ao maior tempo de participação no coral.

Os valores dos parâmetros da avaliação pulmonar pré e pós-treinamento respiratório, dos grupos experimental e controle, bem como cálculo da diferença, podem ser observados na tabela 1 .

Houve significância estatística $(p \leq 0,05)$ em todos os parâmetros avaliados (PImáx, PEmáx, VC, VM) na comparação intragrupo experimental e intergrupos, quando comparados os valores pré e pós-treinamento respiratório realizados pelo grupo experimental, considerando equiparidade dos grupos na avaliação inicial e diferença significativa após o treinamento para os valores do grupo experimental. Na comparação intragrupo controle houve redução dos valores iniciais, porém não significativa estatisticamente.

Os valores dos parâmetros da avaliação vocal pré e pós-treinamento respiratório dos grupos experimental e controle, bem como o cálculo da diferença, estão representados na tabela 2 .

Os dados apresentados referem significância estatística $(p \leq 0,05)$ em todos os parâmetros avaliados (Extensão Vocal, TMF /s/ e TMF/z/) na comparação intergrupos, quando comparados os valores pré e pós-treinamento respiratório realizado pelo grupo experimental, considerando equiparidade dos grupos na avaliação inicial e diferença significativa após o treinamento para os valores do grupo experimental. Na comparação intragrupo experimental, houve significância estatística apenas para os valores de TMF, e, na comparação intragrupo controle, houve redução dos valores iniciais, porém não significativa estatisticamente. 
Quando comparados os grupos experimental e controle, os resultados apontam equiparidade destes na avaliação inicial, ou seja, ambos partiram de um limiar equivalente, sem diferença significativa. Em relação aos valores finais, essa equiparidade é distanciada de forma significativa, com valores positivos para o grupo experimental, após a realização do treinamento respiratório. Tais dados podem ser melhor observados na tabela 3 .

\section{Discussão}

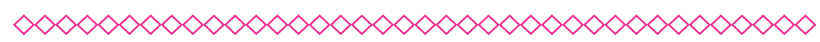

Diversos estudos encontrados na literatura ocuparam-se da avaliação da função respiratória, bem como da função vocal, nos diversos parâmetros abordados nesse estudo, tanto para fins avaliativos quanto para fins comparativos de intervenção terapêutica, seja em populações hígidas, seja em processos patológicos. Porém, quase na totalidade, os estudos não faziam relação entre parâmetros respiratórios e vocais.

Em um estudo com cinco cantores, foi realizado avaliação dos volumes pulmonares, pressões respiratórias máximas e tempo máximo de fonação, pré e pós-treinamento com Ultrabreathe ${ }^{\circledR}$, desenvolvido especificamente para o estudo, realizado todos os dias por dois meses consecutivos. Os resultados demonstraram não haver diferenças significativas nos volumes pulmonares e que houve diferenças significativas nos valores pré e pós-treinamento da PImáx $(62,2 \pm 13,5),(78,2 \pm 3,7)$; PEmáx $(56,4 \pm 7,5),(70,0 \pm 8,1)$; e TMF $(23,2 \pm 4,3)$, $(31 \pm 3,7)^{7}$. Embora utilizando dispositivo de treinamento diferente e apresentando metodologia distinta, esse foi o estudo que mais se assemelhou a presente pesquisa. Entretanto os resultados tenham sido positivos sobre as pressões respiratórias máximas e o TMF, os valores encontrados por essa pesquisa estão aquém dos achados por nosso estudo. Além disso, não foram encontradas diferenças significativas nos volumes pulmonares, fato observado no atual estudo.

Outro estudo encontrou resultados significativos na PImáx de um paciente com esclerose múltipla após aplicação de um protocolo de treinamento com Threshold® IMT, no período de trinta dias, na frequência de três a cinco vezes por semana, por 15 minutos, com cargas a 50\% da PImáx. Os valores da PImáx passaram de $50 \mathrm{cmH}_{2} 0$ no pré-treinamento para $100 \mathrm{cmH}_{2} 0$ na reavaliação após o treinamento ${ }^{8}$. Nesse estudo, observou-se também manutenção dos valores da PEmáx em $120 \mathrm{cmH}_{2} 0$.

A literatura também apresenta diferenças significativas nas pressões respiratórias máximas após aplicação de um protocolo de treinamento utilizando Threshold $\circledast$ IMT com 13 pacientes portadores de fibrose cística. Houve incremento na PImáx que passou de $70 \mathrm{cmH}_{2} 0$ na primeira avaliação para $94,23 \mathrm{cmH}_{2} \mathrm{O}$ após o período de treinamento. Em relação à Pemáx, também houve resultado significativo, passando de $67,31 \mathrm{cmH}_{2} 0$ na primeira avaliação para $78,46 \mathrm{cmH}_{2} 0$ na reavaliação ${ }^{9}$.

Os estudos encontrados relacionando PImáx e PEmáx obtiveram resultados semelhantes aos encontrados por esse estudo quando suas metodologias tratavam-se de protocolos de treinamento com dispositivos diversos. Entretanto, embora com resultados significativos, todos encontraram valores finais das pressões respiratórias máximas inferiores aos observados por esse estudo. Tal fato poderia ser justificado pelas populações de estudo apresentarem determinados comprometimentos com envolvimento pulmonar e esse estudo ter como amostra uma população hígida. Todavia, alguns dos estudos também foram realizados com populações hígidas e também apresentaram resultados inferiores.

Estudos realizados utilizando como parâmetros de avaliação a ventilometria com a análise dos volumes pulmonares demonstraram semelhanças e diferenças com o presente estudo.

Observou-se em uma pesquisa de 44 indivíduos com idades entre 45 e 75 anos, sendo 29 portadores de DPOC e os demais indivíduos hígidos, para estudar o padrão respiratório, movimentação tóraco-abdominal e a ventilação durante a respiração diafragmática. Realizou protocolo de intervenção de 10 minutos, constituídos de 4 minutos de respiração tranquila, 2 minutos de respiração diafragmática e mais 4 minutos de respiração tranquila. Os resultados da avaliação da ventilometria demonstram aumento considerável do volume corrente durante a realização da respiração diafragmática nos grupos controle, estudo moderado e estudo grave, com valores pré-respiração 
diafragmática, durante e após respiração diafragmática (em $\mathrm{ml}$ ), respectivamente, conforme segue: controle (411 / 794 / 374); estudo moderado (384 / 634 / 380); estudo grave (476 / 790 / 471). Tal pequisa também ressalta que a ventilação pulmonar minuto se elevou significativamente durante a respiração diafragmática, com valores correspondentes (em L/min), respectivamente: controle (8 / 14 / 9); estudo moderado (11 / 13 / 11); estudo grave $(10 / 11 / 10)^{10}$.

Esse estudo propõe que o aumento do VC é um importante marcador da eficiência da respiração diafragmática, demonstrando que a associação positiva e significante entre movimento diafragmático e elevação do VC durante a respiração diafragmática sugere que a mobilidade do diafragma pode ser determinante na capacidade de incremento ventilatório.

Em estudo sobre eletroestimulação diafragmática transcutânea em indivíduos saudáveis, vinte mulheres jovens foram divididas em grupo estudo e grupo controle. 0 grupo estudo foi submetido à eletroestimulação suficiente para promover contração muscular e o grupo controle foi submetido à intervenção com intensidade mínima com sensação de formigamento, por dez sessões de trinta minutos. Os resultados no grupo controle não foram significativos, enquanto que no grupo experimental foram significativos as variáveis PImáx, VC e VM ${ }^{11}$.

Quando refere-se a ventilometria e a espirometria, a literatura demonstra que em uma amostra de 13 pacientes com idade média de 43,3 anos, submetidos à cirurgia de hipertensão portal, no pré e pós-operatório (48 horas), os valores foram de $15,12 \mathrm{~L} / \mathrm{min}$ no pré-operatório e de 11,98L/min para VM; sobre os valores do VC, esses foram: 1,04L/min no pré-operatório e 0,60L/min no pós-operatório ${ }^{12}$.

Os valores encontrados nos estudos supracitados tanto do VC quanto do VM são consideravelmente menores dos encontrados nesse estudo, tanto do grupo estudo, quanto do grupo controle. Tal fato pode estar ligado às adaptações fisiológicas do sistema respiratório dos coralistas às exigências da arte do canto, tornando esse sistema mais condicionado em relação à população em geral e, principalmente, em relação aos doentes pulmonares, bem como a realização do treinamento com Threshold $\circledast$ IMT. A constatação da inter-rela- ção entre respiração diafragmática e incremento nos volumes pulmonares também faz ligação ao padrão respiratório dos coralistas, podendo ser um dos indicativos dos resultados encontrados. Além disso, os estudos encontrados apresentaram uma média de idade superior a do presente estudo, o que pode ser um indicativo de volumes pulmonares menores, uma vez que a relação entre idade e volumes pulmonares é inversamente proporcional.

Em relação aos parâmetros da avaliação vocal, a literatura pesquisada também aponta para semelhanças e diferenças a esse estudo.

Ao que se refere à extensão vocal, o número de semitons alcançados pelos sujeitos deste estudo vão ao encontro dos referidos na literatura, os quais enfatizam um mínimo de 20 semitons como medida de extensão vocal normal e passivel de ser produzida por indivíduos com pregas vocais sadias $^{13}$. Os resultados encontrados cobrem essa faixa citada na literatura e são considerados significativos e de ampla importância na prática do canto.

Há também um estudo no qual comparara a extensão vocal de cantores evangélicos com dados oferecidos por literatura já existente. Foram avaliados 80 sujeitos ( 26 homens e 54 mulheres) e os autores concluíram que a extensão vocal média dos participantes foi de 2,5 oitavas, embora tenha-se observado diferença significativa quanto à idade e o tempo de canto no coral. Houve relação estatística significante entre o tempo de experiência e a idade dos cantores, e tempo de participação no coral e número de oitavas alcançadas ${ }^{14}$. Em nosso estudo, não houve correlação com diferença significativa $(p \geq 0,05)$ sobre tempo de participação no coral e faixa etária, visto que o maior percentual da amostra tem apenas um ano de prática do canto, e mesmo os participantes com maior tempo de participação alcançaram resultados semelhantes em todas as variáveis estudadas aos participantes com menor experiência vocal, bem como a faixa etária do estudo foi homogênea, não havendo discrepâncias de valor significativo.

Outros autores mensuraram a extensão vocal de 60 indivíduos falantes normais do português brasileiro e verificaram uma faixa de extensão vocal de 20 semitons a 37 semitons para as participantes do sexo feminino e uma faixa de 20 semitons a 39 semitons para os participantes do sexo 
masculino. Visto que apenas um sujeito de cada gênero alcançou tal extensão, ficando a média em torno de 23 a 28 semitons, o estudo apresentou similaridade com os achados desse estudo ${ }^{1}$.

Os resultados do TMF /s/ e / $/$ / demonstram estar em conformidade com os valores previstos pela literatura de 20 segundos para homens e 14 segundos para mulheres, sendo considerados valores abaixo de 10 segundos como indicativo de patologia vocal com comprometimento respiratório ${ }^{15}$.

Em relação à fricativa /s, 3 sujeitos do grupo encontravam-se abaixo dos valores preconizados na avaliação primeira, visto que todos superaram significativamente o valor previsto no pós-treinamento. Sobre a fricativa /z/, 4 sujeitos do grupo estavam aquém dos valores preconizados na literatura e também superaram significativamente esses após o treinamento com Threshold $\circledast$ IMT. Aos valores do grupo controle, todos os sujeitos apresentaram redução dos parâmetros, com resultado final de 6 sujeitos aquém dos valores preconizados, tanto para fricativa /s/ quanto para /z/. Nenhum participante teve TMF abaixo de 10 segundos, o que indica não haver comprometimento respiratório relacionado ao suporte de ar. Isso demonstra a eficácia do treinamento com Threshold® IMT também nas variáveis correspondentes a função vocal, vista e sacramentada a sua interdependência com o sistema respiratório.

Para se estabelecer valores de referência nos resultados da avaliação do TMF, foi realizado com diferentes gêneros e faixas etárias de falantes do português brasileiro. Os sujeitos do estudo foram compostos por 148 adultos, com idades entre 20 e 49 anos, sem história de problemas neurológicos, distúrbios da comunicação e doenças respiratórias baixas. Os sujeitos foram divididos em grupos por faixa etária de 20-29 anos, 30-39 anos e 40-49 anos, bem como por sexo feminino e masculino. A média dos valores de $/ \mathrm{s} / \mathrm{,} / \mathrm{z} / \mathrm{para}$ as mulheres de $20-29$ anos foram: $(13,48 \pm 5,17)$ e $(13,48 \pm 4,33)$, respectivamente. Para as mulheres de $30-39$ anos os valores foram: $(12,88 \pm 3,43)$ e $(11,92 \pm 3,20)$, respectivamente. Para as mulheres de $40-49$ anos os valores foram: $(12,00 \pm 3,30)$ e $(11,36 \pm 3,41)$, respectivamente. Para os homens de $20-29$ anos os valores foram: $(18,56 \pm 8,56)$ e $(20,32 \pm 9,25)$, respectivamente. Para os homens de 30-39 anos os valores foram: $(14,78 \pm 5,89)$ e $(16,30 \pm 5,93)$, respectivamente. Para os homens de 40-49 anos os valores foram: $(15,04 \pm 6,68)$ e $(15,76 \pm 7,07)$, respectivamente ${ }^{16}$.

Os resultados encontrados por esse estudo demonstram que os valores preconizados pela literatura nem sempre são realidade junto aos sujeitos estudados, pois para todas as variáveis, exceto /z/ de homens na faixa etária de 20-29 anos, estão abaixo dos valores de referência. Esses valores demonstram-se abaixo dos valores encontrados por esse estudo, tanto nos tempos pré como pós-treinamento, nos grupos estudo e controle. Tal fato pode ser justificado pelo grupo estudado se tratar de uma população em específico, com resultados relacionados também a prática do canto como fator importante no desenvolvimento da função pulmonar.

Estudo realizado por Vicco e Behlau ${ }^{17}$ onde avaliaram o perfil vocal de 27 cantores brasileiros de Karaoke, com idade entre 16 e 68 anos, divididas por gênero, os valores de TMF /s/ de foram de: 24 segundos para homens e 18 segundos para mulheres; valores de TMF / z/ de: 20 segundos para homens e 17 segundos para mulheres. Os resultados encontrados por esse estudo foram menores dos resultados encontrados no grupo estudo e maiores dos resultados no grupo controle.

Em outro estudo com uma amostra de 20 cantores com idade entre 17 e 39 anos, analisando as variáveis TMF /s/ e /z/ comparando valores pré e pós-ensaio. Dos resultados encontrados, pré e pós-ensaio, respectivamente, foram: TMF /s/ (28 para 30 segundos); TMF /z/ (26 para 28 segundos) ${ }^{18}$. Tais resultados pós-ensaio demonstram valores menores e equiparados aos resultados finais encontrados pelo grupo estudo e maiores dos resultados encontrados pelo grupo controle em relação ao TMF $/ \mathrm{s} / \mathrm{e} / \mathrm{z} /{ }^{18}$. Vale salientar que as avaliações da atual pesquisa foram realizadas todas pré-ensaio, sem aquecimento vocal e/ou respiratório.

Os estudos realizados por outros autores correlacionados ao presente texto, bem como os dados encontrados na literatura, demonstraram semelhanças e diferenças com o presente. Por meio dessa pesquisa, encontraram-se resultados positivos nas variáveis analisadas quando comparados grupo estudo e controle pré e pós-treinamento, demonstrando eficácia da metodologia adotada. 
Além disso, alguns parâmetros já se encontravam com valores elevados, inferindo uma população de estudo com adaptações relacionadas ao canto.

\section{Considerações finais}

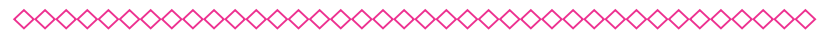

0 treinamento respiratório (de 15 intervenções) com o uso do incentivador Threhold $®$ IMT mostrou-se eficaz no fortalecimento muscular respiratório. Possibilitou incremento de volumes pulmonares e nas funções vocais de extensão, tempo máximo de fonação das fricativas /s/ e /z/, direta e indiretamente, bem como na normalização da relação $s / z$, refletindo em uma nova possibilidade de treinamento junto aos cantores/coralistas para o melhor desempenho da arte do canto.

Porém, este modo terapêutico de treinamento não é muito difundido neste meio e o encargo financeiro para aquisição deste incentivador são fatores que podem limitar esta prática.

Desta forma, sugere-se a realização de outros estudos com amostras maiores como também com outras populações e equipamentos. Assim permitiria viabilizar a comparação e ainda correlacionar com o uso do Threhold $\circledast$ IMT em treinamentos respiratórios.

\section{Referências}

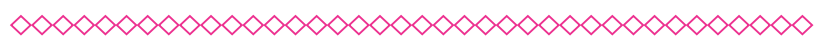

1. Vargas, AC. et al. Perfil de Extensão Vocal em Indivíduos Falantes Normais do Português Brasileiro. Cefac. 2005;7(1):108-116.

2. Pinho, SMR. Fundamentos em Fonoaudiologia: Tratando os Distúrbios da Voz. 2. ed. Rio de Janeiro: Guanabara Koogan, 2003.

3. Quinteiro, EA. Estética da Voz. 5. ed. São Paulo: Plexus, 2007.

4. Costa, D. Fisioterapia Respiratória Básica. 1. ed. São Paulo: Atheneu, 2004.

5. Deturk, WE; Cahalin, LP. Fisioterapia

Cardiorrespiratória. Porto Alegre, Artmed, 2007.
6. Pinho, SMR.; Bastos, PRJ. Quadro para Avaliação Vocal de correspondentes Tonais. Barueri: Pró-Fono, 2003.

7. Nam, DH. Specially Programmed Respiratory Muscle Trainig for Singers by Using Respiratory Muscle Trainig Device (Ultrabreathe $\left.{ }^{\circledR}\right)$. Yonsei Medical Journal 2004;45(5):810-817.

8. Dalsasso, GC. Treinamento Muscular Respiratório em um Paciente com Esclerose Múltipla: Estudo de Caso. 2007. 98f. Monografia (Bacharel em Fisioterapia) - Faculdade Assis Gurgacz, Cascavel, 2007.

9. Galvão, F. Avaliação dos Efeitos do Treinamento Muscular Inspiratório em Pacientes com Fibrose Cística através do Uso do Threshold IMT. 2006. 88f. Dissertação (Mestrado em Saúde da Criança e do Adolescente) - Faculdade de Ciências Médicas, Universidade Estadual de Campinas, Campinas, 2006.

10. Fernandes, M. Estudo do Padrão Respiratório, Movimentação Toracoabdominal e Ventilação em Pacientes Portadores de Doença Pulmonar Obstrutiva Crônica Durante Respiração Diafragmática. 2007. 112f. Tese (Doutorado em Ciências) - Faculdade de Medicina, Universidade de São Paulo, São Paulo, 2007.

11. Forti, EM. et al. Eletroestimulação diafragmática transcutânea em indivíduos saudáveis. Fisioterapia Brasil. 2005; 6(4): 261-264.

12. Araújo Filho, AA. Avaliação da Ventilometria e Espirometria no Pré e Pós-Operatório de Pacientes Submetidos à Cirurgia de Hipertensão Portal. 2007. 81f. Dissertação (Mestrado em Ciências da Saúde). Universidade Federal de Sergipe, Aracaju, 2007.

13. Bonne, D. McFarlane, S. A voz e a Terapia Vocal. Porto Alegre: Artes Médicas, 1994.

14. Costa, PJBM. et al. Extensão Vocal de Cantores de Coros Evangélicos Amadores. Cefac. 2006; 8(1):96-106.

15. Behlau, M; Pontes, P. Avaliação e Tratamento das Disfonias. São Paulo: Lovise, 1995. 
16. Beraldinelle, R. et al. Tempo Máximo de Fonação em Adultos. Anais Bauru FOB-USP. São Paulo, p. 98. 2007.

17. Vicco, DC; Behlau, M. Perfil Vocal de Cantores Brasileiros de Karaoke. In: Behlau, M. Gasparini, G. A Voz do Especialista. Vol. I. Rio de Janeiro: Revinter, 2001.
18. Torres; MLGM; Behlau, M. Medidas do Perfil de Extensão Vocal, Tempo Máximo de Fonação e Relação s/z em Cantores de um Coral Amador Pré e Pós-Ensaio. In: Behlau, M. Gasparini, G. A Voz do Especialista. Vol. I. Rio de Janeiro: Revinter, 2001.

\section{Anexos}

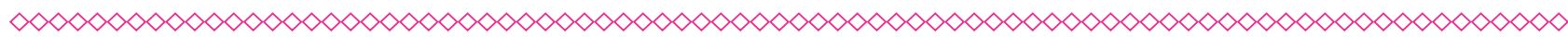

Tabela 1 - Avaliação da Função Pulmonar

\begin{tabular}{|c|c|c|c|c|}
\hline Variável & Grupo & Pré-treinamento & Pós-treinamento & Diferença (pós-pré \\
\hline PImáx & Estudo & $81,25 \pm 6,40$ & $120 \pm 0,00$ & 38,75 \\
\hline \multirow[t]{2}{*}{$(\mathrm{cmH} 20)$} & Controle & $64,37 \pm 14,98$ & $56,25 \pm 18,46$ & $-8,12$ \\
\hline & & & & $\begin{array}{l}\text { Valor corrigido } \\
46,87\end{array}$ \\
\hline PEmáx & Estudo & $65,62 \pm 11,78$ & $120 \pm 0,00$ & 54,38 \\
\hline \multirow[t]{2}{*}{$(\mathrm{cmH} 20)$} & Controle & $56,87 \pm 17,10$ & $55 \pm 14,14$ & $-1,87$ \\
\hline & & & & $\begin{array}{l}\text { Valor corrigido } \\
56,25\end{array}$ \\
\hline VC & Estudo & $3,33 \pm 1,31$ & $4,59 \pm 1,04$ & 1,26 \\
\hline \multirow[t]{2}{*}{ (L/min) } & Controle & $3,07 \pm 7,50$ & $2,34 \pm 3,94$ & $-0,73$ \\
\hline & & & & $\begin{array}{c}\text { Valor corrigido } \\
1,99\end{array}$ \\
\hline VM & Estudo & $29,24 \pm 22,77$ & $35,76 \pm 21,83$ & 6,52 \\
\hline \multirow[t]{2}{*}{ (L/min) } & Controle & $21,09 \pm 10,76$ & $16,85 \pm 7,24$ & $-4,24$ \\
\hline & & & & $\begin{array}{c}\text { Valor corrigido } \\
10,76\end{array}$ \\
\hline
\end{tabular}

Legenda: Valores expressos em média e desvio padrão correspondentes ao pré e pós-treinamento e cálculo da diferença do grupo estudo e controle de todas as variáveis da avaliação da função respiratória.

Fonte: elaboração dos autores.

(clique para voltar ao texto) 
Tabela 2 - Avaliação da Função Vocal

\begin{tabular}{llrrc} 
Variável & Grupo & Pré-treinamento & Pós-treinamento & Diferença (pós-pré) \\
Extensão & Estudo & $23,12 \pm 3,60$ & $27,37 \pm 1,50$ & 4,25 \\
Vocal (st) & Controle & $23,12 \pm 3,68$ & $20,25 \pm 3,01$ & $-2,87$ \\
& & & $\begin{array}{c}\text { Valor corrigido } \\
\end{array}$ \\
& & & 7,12 \\
& & & 10,75 \\
TMF: /s/ & Estudo & $22,75 \pm 7,20$ & $33,50 \pm 7,17$ & $-2,63$ \\
(seg.) & Controle & $19,25 \pm 5,44$ & $16,62 \pm 3,20$ & Valor corrigido \\
& & & 13,38 \\
& & & 10,50 \\
\hline TMF: /z/ & ESTUDO & $18,25 \pm 5,25$ & $28,75 \pm 6,51$ & 1 \\
\hline
\end{tabular}

Legenda: Valores expressos em média e desvio padrão correspondentes ao pré e pós-treinamento e valores de diferença do grupo estudo e controle das variáveis da avaliação da função vocal.

Fonte: elaboração dos autores.

(clique para voltar ao texto)

Tabela 3 - Categorização dos Valores de Significância Pré e Pós-Treinamento

\begin{tabular}{lcc} 
Variável & Pré-treinamento & Pós-treinamento \\
PImáx & $(p=0,1)$ & $(p<0,0001)^{*}$ \\
PEmáx & $(p=0,25)$ & $(p<0,0001)^{*}$ \\
VC & $(p=0,64)$ & $(p=0,0005)^{*}$ \\
VM & $(p=0,37)$ & $(p=0,04)^{*}$ \\
Extensão Vocal & $(p=1,00)$ & $(p<0,0001)^{*}$ \\
TMF/s/ & $(p=0,29)$ & $(p<0,0001)^{*}$ \\
TMF/z/ & $(p=0,74)$ & $(p=0,0008)^{*}$ \\
Relação s/z & $(p=0,02)$ & $(p=0,02)^{*}$ \\
\hline
\end{tabular}

Legenda: Categorização dos Valores do Grupo Estudo e Controle, e na comparação Inter-Grupos (Significância $p \leq 0,05$ ).

*Valores Significantes.

Fonte: elaboração dos autores. 